\title{
Fabian Dombrowski"
}

\section{Profit First - Safety Second!?**}

Flugsicherheit als deregulierte Funktion der Luftfahrtunternehmen - Eine empirische Studie

Hintergrund des vorliegenden Beitrags ist die fortschreitende Deregulierung der Flugsicherheit, die entsprechend stärker in den Verantwortungsbereich der Luftfahrtunternehmen übertragen wird und damit potenziell ökonomischen Kosten-Nutzen-Kalkülen unterworfen werden kann. Im Mittelpunkt steht die Frage, wie Luftfahrtunternehmen mit ihren elementaren Zielsetzungen Flugsicherheit und Profitabilität umgehen. Dieser praxisrelevanten Problemstellung wird vermittels einer empirischen Studie nachgegangen.

Schlagwörter: Airline Safety Management System, Deregulierung, Profitabilität, Unternehmensethik

\section{Profit First - Safety Second!? Flight Safety as a Deregulated Function of Airline Companies - an Empirical Study}

The background of this article is the progressing deregulation of flight safety, which by consequence becomes more and more a responsibility of airline companies themselves. As a result it might become subject to economic cost-benefit calculations. The central question is how airline companies deal with their two fundamental objectives flight safety and profitability. An empirical study helps to examine this practical problem.

Keywords: Airline Safety Management System, Deregulation, Profitability, Business Ethics

\section{Einleitung}

Fliegen ist schon lange nicht mehr einer exklusiven Gesellschaftsschicht vorbehalten. Billigangebote gibt es mittlerweile von fast allen Fluggesellschaften, wobei deren Preise von manchen Beobachtern tatsächlich als zu günstig eingeschätzt werden (vgl. Busse 2013). Nichtsdestotrotz gilt das Flugzeug als das derzeit sicherste Verkehrsmittel - auch wenn man zuweilen von Vorfällen hört, bei denen Luftfahrtunternehmen auf Kosten der Sicherheit sparen (vgl. RTE 2012) und man im Internet Kundenberichte über Sicherheitsprobleme bei Airlines finden kann (vgl. Ryanair-Safety 2013). In dieser Situation wird nun - quasi im Gefolge des

* Fabian Dombrowski, B.Sc. Wirtschaftswissenschaften, Verkehrsflugzeugführer, E-Mail: fabian_dombrowski@web.de, Forschungsschwerpunkte: CSR, Unternehmensethik, Airline Safety Management System, Deregulierung. Mein ganz besonderer Dank gilt Dr. Thomas Kuhn für eine wunderbare Unterstützung. Außerdem danke ich Raphaela Bardutzky, Patrick Reisch und den anonymen Gutachtern für kritische Anregungen.

* Beitrag eingereicht am 31.10.2014; nach doppelt verdecktem Gutachterverfahren überarbeitete Fassung angenommen am 01.02.2017. 
neoliberalen Deregulierungstrends (vgl. Butterwegge et al. 2008; Canedo 2008) die Aufsicht über Flugsicherheit grundlegend verändert (vgl. ICAO 2013). Flugsicherheit soll zukünftig stärker in Eigenverantwortung der Fluggesellschaften verwirklicht werden - Fluggesellschaften, die im globalen Wettbewerb versucht sein könnten, an Sicherheit zu sparen. Kann in der Folge dieser Deregulierung die Flugsicherheit auch weiterhin gewährleistet werden oder wird sie zum Spielball anderer Ziele?

Zur Diskussion dieser Fragestellung wird zunächst die Neuregulierung der Flugsicherheitsaufsicht genauer dargestellt, die damit gleichsam als ökonomisches Kosten-Nutzen-Kalkül neu interpretiert werden kann. Diese Sichtweise wird mit der >Harmonie $<$ - und `Konfliktthese` der Unternehmensethik hinterlegt, die als theoretische Referenz für eine empirische Untersuchung dienen, welche Einschätzungen von Experten über den Zusammenhang zwischen Flugsicherheit und wirtschaftlichem Erfolg aufzeigt. Hierdurch kann zuletzt genauer eingeschätzt werden, welche Auswirkungen die regulatorische Neuausrichtung von Flugsicherheit zeitigen könnte.

\section{Safety Management System (SMS): Flugsicherheit unter der Ägide der Deregulierung}

Über weite Teile des 20. Jahrhunderts herrschte die Überzeugung, dass staatliche Behörden die Wettbewerbsbedingungen umfassend regulieren müssen, damit sich die Luftfahrt zum Wohle der gesamten Gesellschaft entwickeln kann (vgl. Schenk 2004: 10f., 60). Andernfalls galt ein flächendeckendes Streckennetzwerk als unwahrscheinlich, ruinöse Konkurrenz hingegen als sehr wahrscheinlich (vgl. Button 1991: 6). Im Laufe der Zeit wurde dabei jedoch eine Überbürokratisierung mit einem uneinheitlichen Regelwerk festgestellt und gleichzeitig bemängelt, dass Luftfahrtunternehmen Gesetzeslücken auf Kosten der Sicherheit ausnutzten (vgl. Berkeley et al. 2008: 11ff.).

Überdies machten fehlende Ressourcen der Behörden eine Überwachung der strenger werdenden Regeln zusehends schwieriger. Systematische Überwachung wurde deshalb durch Ad-hoc-Kontrollen ersetzt. Bezeichnenderweise konnten alle vorgeschriebenen Inspektionen erst abgearbeitet werden, als man einen Großteil der Vorschriften strich. Auch kam Kritik auf, dass die Behörden entscheidende Sicherheitstrends verpassten. In Anbetracht immer größer werdender Anforderungen an die staatliche Flugsicherheitsaufsicht und um eigenen Kostensteigerungen Einhalt zu gewähren, übertrugen die Behörden Verantwortung zusehends an die Luftfahrtunternehmen trotz durchaus schlechter Erfahrungen (vgl. ebd.).

Infolge zunehmender Interdependenzen in der Luftfahrt, bei gleichzeitig immer verlässlicherer Technik, avancierten menschliche und operationelle Gesichtspunkte zur primären Gefährdung der Sicherheit. Das Regelsystem wurde seinem ursprünglichen Ziel, Flugsicherheit zu steigern, nicht mehr gerecht (vgl. Harris 2011). Wie Berkeley et al. (2008: 37) zeigen, hat sich im Laufe der Geschichte die Flugsicherheitsaufsicht von einer ’hybrid craft/coping«-Behörde in eine >procedu- 
ralk-Organisation gewandelt. Also von einer Behörde, die kaum beobachten kann, was für Flugsicherheit getan wird, und nur teilweise die Auswirkungen auf den Sicherheitsstandard erkennen kann, hin zu einer Organisation, die zwar beobachten kann, was für Flugsicherheit getan wird, aber nur schwer die Wirksamkeit dieser Maßnahmen zu beurteilen vermag - da Vorfälle in der kommerziellen Luftfahrt selten geworden sind. Entsprechend wird versucht, quantitative Verbindungen zwischen Gefahren und Vorfällen herzustellen, um Flugsicherheitsaufsicht wie eine Art Produktionseinheit >managen`zu können (ebd.).

Um sich der in der Luftfahrt ständig ändernden Umwelt anzupassen, bedarf es nach Harris (2011) einer systemischen Betrachtung. Der Autor resümiert, dass zzerstückelte Regularien - ohne systemweite Perspektive - die Sicherheit nicht wie gewünscht steigern könnten. Außerdem würden starre Regeln den technologischen Fortschritt sowie den Flugbetrieb selbst behindern (ebd.). Mit dem International Civil Aviation Organization (ICAO) Document (DOC) 9859 (vgl. ICAO 2013: 1-1) wird nun die Umsetzung eines Safety Management System (SMS) für Luftfahrtunternehmen vorgeschrieben. Dabei wird Sicherheit als Führungsaufgabe definiert (ebd.: 2-10). Klompstra et al. (2012) gehen davon aus, dass hiermit Verantwortung für Sicherheit auf allen Ebenen einer Organisation übernommen wird, wodurch die Sicherheit insgesamt verbessert wird. Die Rechtsgrundlage für deutsche Luftfahrtunternehmen zur Einführung eines Safety Management Systems ergibt sich durch Maßgabe des Bundesministeriums der Justiz (2008). Danach muss ein >Acceptable Level of Safety Performancer (ALoSP) gewährleistet sein, der wie folgt definiert wird: » The minimum level of safety performance (...) of a service provider, as defined in its safety management system, expressed in terms of safety performance targets and safety performance indicators " (ICAO 2013: xii). Im Rahmen dessen darf der akzeptable Sicherheitsstandard (ALoSP) sogar unterschritten werden - als sog. tolerabler Sicherheitslevel, unter der Voraussetzung begleitender Risiko-Abschwächungsmaßnahmen (vgl. ICAO 2013: 2-30f.).

Flugsicherheit besteht demnach, sobald die Wahrscheinlichkeit, dass Personen Schaden zugefügt wird, kleiner oder gleich dem ALoSP ist. Systemisch sollen nicht nur sogenannte raktive Fehler der Individuen aus der operationellen Umgebung durch etwa entsprechende Arbeitsbedingungen beachtet werden, sondern auch sogenannte >latente Fehler', wie falsche Management-Entscheidungen oder konfligierende Ziele innerhalb der Organisation. Solch latente Fehler sollen erkannt und durch geeignete Abwehrmaßnahmen vermieden werden (vgl. ebd.). Die letztendliche Verantwortung für den Sicherheitsstandard eines Luftfahrtunternehmens liegt somit beim Management, denn organisationale Faktoren befinden sich außerhalb des Blickfeldes traditioneller Flugsicherheitsaufsicht. "Safety risk management is therefore a key component " (ebd.: 2-30): »there are no fixed (mandatory) prescribed safety indicators or alert levels or prescribed values " (ebd.: 2-32). So kann der Sicherheitsstandard einer Fluggesellschaft zuletzt entsprechend ihrer - sich selbst gestellten - Anforderungen angepasst werden.

Das Aufsichtssystem bestand bisher somit aus Regeln, die die Behörde beschlossen hatte, weil sie glaubte, dass diese zu einer hohen Flugsicherheit führen würden. Die Behörde überwachte die Unternehmen und setzte damit die Einhaltung 
der Regeln durch (vgl. Berkeley et al. 2008: 27). Zukünftig sollen sich die Luftfahrtunternehmen weitgehend selber beaufsichtigen, sodass die Flugsicherheit zu einer Management-Funktion wird. Reason (1990: 476, eigene Hervorhebung), auf den die Fundamente des SMS zurückgehen (vgl. ICAO 2013: 2ff.), erklärt dazu: »Further improvements in reliability will require more effective methods of risk management. «

\section{Flugsicherheit als ökonomisches Kosten-Nutzen-Kalkül}

Cokorilo et al. (2010) sehen die Aufgabe von Risk Management im Rahmen eines SMS in der Beseitigung von Gefahren, die das Überleben einer Organisation bedrohen. Wobei es aber unrealistisch oder wirtschaftlich nicht praktikabel ist, tatsächlich alle Risiken zu eliminieren. Mit dem quantitativen Werkzeug einer >cost-benefit analysis` müssen die Unfallwahrscheinlichkeit und die damit verbundenen Aufwendungen mit den Kosten für die Maßnahmen einer gesteigerten Flugsicherheit ausbalanciert werden (vgl. ebd.: 199; vgl. ferner ICAO 2013: 2-21). Damit ist selbstverständlich auch das anzustrebende Niveau der Flugsicherheit eines Luftfahrtunternehmens durch Kosten beeinflusst. Cokorilo et al. (vgl. 2010: 193ff.) scheuen sich in diesem Zusammenhang nicht, Menschenleben einen Preis zu geben, der zudem nach geographischen Regionen variiert. ${ }^{1}$

Chacin (2013) betont die Wichtigkeit eines quantitativen Ansatzes für die Allokation von Ressourcen, weil man so besser argumentieren und Entscheidungen verteidigen kann. Im International Civil Aviation Organization (ICAO) Document (DOC) 9859 (2013: 4-13) wird schließlich erläutert, wie man quantitative Sicherheitsziele eines Luftfahrtunternehmens mit einer >basic MS Excel function< setzt wobei eine volatilere historische Datenbasis großzügigere salert levels in der folgenden Periode zulässt.

Luftfahrtaufsichtsbehörden sind traditionell mit der Schwierigkeit konfrontiert, sowohl sicherheitsrelevante als auch wirtschaftliche Ziele zu fördern und dabei ihre eigenen Kosten im Blick zu halten (vgl. Berkeley et al. 2008: 34). Aufgrund dessen vertrauten die Aufsichtsbehörden oftmals darauf, dass Luftfahrtunternehmen sich freiwillig an bestehende Vorschriften halten. Auch wurden Regulierungen abgewendet, um wirtschaftliche Härten zu vermeiden. Jedoch führten solche 'Gentlemen's Agreements` zuweilen zu Katastrophen, da sich die Fluggesellschaften eben nicht hieran hielten (vgl. ebd.: 23). Die New York Times kommentierte hierzu einst: »(N)ot necessarily outright bribery and graft, but the pervasive corruption of advantage, deception, greed and disregard for the public interest « (Wicker 1976). Lediglich akkordierte Vereinbarungen unter den Fluggesellschaften schienen zu funktionieren. Wie etwa die, die es aus einem wettbewerblichen Standpunkt heraus erleichterte, Flüge bei schlechtem Wetter ausfallen zu lassen (vgl. Berkeley et al. 2008: 8).

1 Schon innerhalb Europas unterscheidet sich der angenommene durchschnittliche Preis für ein Menschenleben um bis zum mehr als Vierfachen: Dieser >value of statistical life reicht in Europa je nach Land von 650.000 Euro bis zu 2,64 Millionen Euro. 
Korrespondierend hiermit diagnostizieren Raghavan/Rhoades (vgl. 2005: 286) einen grundlegenden Trend, der mit der Deregulierung der Luftfahrtbranche seit 1978 einhergeht: Wachstum rangiert vor Sicherheitsverbesserungen! Die Datenbasis ihrer Untersuchung umfasst die Jahre 1955 bis 2002. Insbesondere die Zeiträume vor und nach 1978 wurden hier auf konvergente bzw. divergente Unfallraten analysiert. Wie es Graham/Bowes (vgl. 1979) bereits befürchteten, kann Kostendruck dazu führen, Wartungsarbeiten mit zum Teil katastrophalen Folgen aufzuschieben (vgl. NTSB 2002: 180). Diese These steht allerdings im Gegensatz zur Einschätzung von Adrangi et al. (vgl. 1997): Sie erwarteten nämlich steigende Sicherheit in liberalisierten Märkten, da Luftfahrtunternehmen daran interessiert seien, das Kundenvertrauen zu sichern. Der Sicherheitsstandard eines Luftfahrtunternehmens wird jedenfalls durch verschiedene Faktoren innerhalb des Unternehmens, wie etwa Wartung, Ausbildung und Ausrüstung beeinflusst (vgl. Rose 1990). Auch die Arbeitsbedingungen des Personals, organisationale Veränderungen, Zeitdruck und ein Ausbalancieren von 'value added « und ssafety risk ‘ beeinflussen den Sicherheitsstandard (vgl. ICAO 2013: 2-13). Steigende Flugsicherheit ist demnach möglich, wenn sie zu einem Wert an sich innerhalb der Organisation wird (ebd.).

Flugsicherheit hängt somit ganz wesentlich vom Top-Management selbst ab (vgl. Chen et al. 2010). Luftfahrtunternehmen können flexibel entscheiden: Über ihre Investitionen und ihr Sicherheitslevel, ebenso über ihren relativen Zugang zu Kapitalmärkten - was wiederum ihre Unfallraten beeinflusst (vgl. Dionne et al. 1997).

Seit der Deregulierung arbeitet die Luftfahrtindustrie in einem weltweit sehr uneinheitlichen Markt, der zusehends gewinnorientierter und enger mit der Privatwirtschaft verflochten ist (vgl. Pompl 2007: 347ff.). Der Fokus der Flugsicherheit hat sich auf die in den Luftfahrtunternehmen liegenden Strukturen verschoben. »The majority [of root causes of significant unsafety events] had their origins in (...) managerial domains « (Reason 1990: 477). Deswegen verantworten Unternehmensführungen neben der wirtschaftlichen Erfolgssicherung auch zunehmend die Gewährleistung von Flugsicherheit. Werden Luftfahrtunternehmen Erfolg und Sicherheit gleichberechtigt nebeneinander verfolgen, oder wird im Zweifel eines der Ziele vorgezogen? Beziehungsweise ist der Konnex doch eher der, dass kein Luftfahrtunternehmen (strategisch) erfolgreich sein kann, das die (maximale) Sicherheit vernachlässigt? Um diese divergenten Einschätzungen theoretisch fundierter beurteilen zu können, soll im Folgenden auf einige korrespondierende Erkenntnisse der Debatte über Unternehmensethik eingegangen werden.

\section{Harmoniethese versus Konfliktthese: Zwei Grundverständnisse von Unternehmensethik}

Darüber, was Unternehmensethik genau ist, gibt es unterschiedliche Vorstellungen. Allgemein formuliert bemüht sich Unternehmensethik darum, normative Konzepte guter Unternehmensführung herauszuarbeiten (vgl. Thielemann/Ulrich 
2009). Zentral ist dabei in der Regel die Frage, wie ein Unternehmen mit seinen Anspruchsgruppen und deren Zielsetzungen verantwortbar umgeht. Diesbezüglich können zwei divergente Grundüberzeugungen unterschieden werden: Zum einen die, dass ein verantwortungsbewusster Umgang mit allen Anspruchsgruppen, ebenso wie ein ethisches Verhalten des Unternehmens, immer auch diesem selbst bzw. dessen wirtschaftlichen Zielen (Gewinnmaximierung) dient (>Harmoniethese`) (vgl. ebd.). Dem steht die Anschauung gegenüber, dass ethische Verhaltensweisen (zum Wohle ihrer Stakeholder) dem Unternehmen durchaus Geld kosten können, das Streben nach Gewinnmaximierung und das Streben nach gesellschaftlicher Verantwortung insofern nicht per se identisch sind (`Konfliktthese $<$ ) (vgl. ebd.).

Die Harmoniethese widerspiegelt sich archetypisch in Milton Friedman's (1970) Diktum: »The social responsibility of business is to increase its profits «. Dahinter steht die Überzeugung, dass Profite durch die sunsichtbare Hand des Marktes ein Ausdruck, der auf Adam Smith zurückgeht - stets gerecht verteilt werden. Albach (vgl. 2005) kommt in Entsprechung dessen zu dem Schluss, dass es schlicht überflüssig sei, sich mit Unternehmensethik zu beschäftigen. Denn Betriebswirtschaftslehre ist bereits Unternehmensethik, da sie aufzeigt, wie ein Unternehmen seinen Kapitalwert maximieren kann.

Die Harmoniethese vergegenwärtigt sich in der aktuellen Debatte über Unternehmensethik v.a. im sogenannten Business Case for Corporate Social Responsibility (CSR) (vgl. Kurucz et al. 2008; Leisinger 2008; Carroll/Shabana 2010). Dieser vertritt die Einschätzung, dass sich Ethik für Unternehmen langfristig auszahlt, wohingegen unethische Verhaltensweisen (Corporate Social Irresponsibility) sich eher früher als später negativ auf den Unternehmenserfolg auswirken werden.

Die supponierte positive Korrelation zwischen Ethik und Erfolg wird gemeinhin mit spezifischen (vor-/ökonomischen) Erfolgswirkungen von CSR-Maßnahmen erklärt (z.B. Reputationsaufbau und Risikoabbau, Umsatzsteigerung und Kostensenkung; vgl. auch Hansen/Schrader 2005), die auf eine 'Belohnung verantwortungsbewusster Unternehmen durch ihre Stakeholder hinauslaufen - wohingegen gesellschaftlich verantwortungslose Unternehmen mit sbestrafenden< Reaktionen ihrer Anspruchsgruppen zu rechnen haben (vgl. Kuhn/Weibler 2011).

Staatliche Regulierung ist damit für >Corporate Social Performance< nicht erforderlich, ebenso wenig wie Integrität des Managements oder ein Handeln aus Pflicht (vgl. Orlitzky et al. 2003: 409). Es kann also der eigene Nutzen maximiert werden, weil der Markt für verantwortungsvolles Handeln sorgt. Unterschiedliche Ansprüche werden demgemäß mit hoher Wahrscheinlichkeit freiwillig ausbalanciert, »based on managers' cost-benefit analysis of a firm's investments « (ebd.: 424f.). Themenbezogen bedeutet dies im Kern: Hohe Flugsicherheit wird gewünscht und deshalb belohnt! Und wer meint, hieran sparen zu können, der wird `von den Märkten abgestraft werden.

Die Konfliktthese geht demgegenüber davon aus, dass gesellschaftliche Verantwortung und wirtschaftlicher Erfolg nicht immer und überall in Einklang stehen. Vertreter dieser Anschauung stehen dem Business Case for CSR entsprechend kritisch gegenüber, sprich: bezweifeln nicht nur dessen empirische Evidenz (vgl. 
Vogel 2006; Kuhn/Weibler 2011; Fleming/Jones 2013), sondern auch dessen ethische Qualität (vgl. Thielemann 2009: 138ff.; Ulrich 2016: 453ff.) und deuten ihn als >Ideologie` (vgl. Banerjee 2007) oder >Mythos`, der über triviale Lehren des Volksmund (»Ehrlich währt am längsten «; »Lügen haben kurze Beine«) kaum hinausreicht (vgl. Raith 2013: 114).

In inhaltlicher Quintessenz besagt die Konfliktthese, dass es zwar durchaus 'Schnittmengen zwischen Erfolg und Ethik (`win-win`zones) geben kann, es jedoch nichtsdestotrotz realiter weithin von (Macht-)Konstellationen auszugehen ist, in denen ethische Verhaltensweisen die Gewinne schmälern und/oder unethische Verhaltensweisen die Profitabilität (auch dauerhaft) steigern (vgl. Haigh/ Jones 2006; Karnani 2010; Kuhn/Weibler 2016). Dem General-Motto des Business Case: ‘Ethics pays! wird mithin differenzierend entgegen gehalten: »Being good doesn't always pay« (Zadek 2004: 6), »It pays to be bad « (Monk 2006) oder auch: »It pays to be good but not too good « (Mintzberg 1983: 10).

Entsprechend diesen Überlegungen, die in der Unternehmensethik-Debatte notabene zunehmend Verbreitung und Anerkennung zu finden scheinen, ist CSR also weniger ein Business Case als vielmehr ein Moral Case zu verstehen (vgl. zum Begriff: Hansen 2010; Raith 2013). Ganz im Sinne der philosophischen Ethik geht es bei moralischen Entscheidungen in Unternehmen demnach um Abwägungen zwischen verschiedenen Gütern, womit sich die prinzipielle Verfolgung eines einzigen Zieles (Gewinnmaximierung) gleichsam verbietet (vgl. auch Steinmann/ Löhr 1991; Ulrich 2016). Themenbezogen bedeutet dies: Höhere Flugsicherheit kann durchaus zu niedrigeren Gewinnen führen - ebenso wie sukzessive Abstriche an der Flugsicherheit zu höheren Gewinnen beitragen können. Und dieses gilt natürlich umso mehr, je weitgehender derlei Güterabwägungen von der politischsystemischen Ebene via Deregulierung auf die institutionelle Ebene des einzelnen (Luftfahrt-)Unternehmens überantwortet werden.

\section{Forschungsfrage und Forschungsmethode}

Überführen wir diese allgemeinen Überlegungen auf unsere spezifische Fragestellung, dann zeigt sich: In der Luftfahrtbranche wird in mannigfaltigen Bereichen Deregulierung vorangetrieben (vgl. ICAO 2013: 2-32; Pompl 2007: 413ff.). Hieraus ergibt sich für die Luftfahrtunternehmen ein neu entstandener Handlungsspielraum bezüglich der eigenen Sicherheitsstandards. Aus der Perspektive der Flugsicherheitsaufsicht erfolgt diese Deregulierung unter anderem mit dem Ziel steigender Flugsicherheit (vgl. Berkeley et al. 2008: 27; ICAO 2013: 5-1) - also quasi aus ethischen Erwägungen heraus. Von praktischer Bedeutung ist allerdings, ob diese Erwartung in einem (zunehmend) deregulierten und wettbewerblichen Markt (vgl. Pompl 2007: 347ff.) realistisch ist.

Zur genaueren Untersuchung dessen dient unsere empirische Studie, die insbesondere folgender Forschungsfrage nachgeht: Stehen Flugsicherheit und wirtschaftlicher Erfolg in einem harmonischen Verhältnis zueinander, oder geraten sie 
im Zuge weiterer Deregulierungen bei hohem Wettbewerbs- und Erfolgsdruck potenziell in Konflikt?

Systematisch wird im Weiteren wie folgt vorgegangen: Zunächst erfolgt eine nähere Analyse formaler Verlautbarungen von Luftfahrtunternehmen (z.B. Geschäftsberichte, Internetauftritte, Publikationen) zu dieser Problematik. Im Anschluss daran widmen wir uns einem gesetzgebenden Prozess im europäischen Parlament zur Regelung von maximalen Flugdienstzeiten für Flugzeugbesatzungen.

Schließlich wenden wir uns dem Inneren der Luftfahrtunternehmen zu, um unsere Erkenntnisfrage aus einer weiteren Perspektive zu betrachten: Wie wird der Zusammenhang Flugsicherheit und wirtschaftlicher Erfolg im betrieblichen Alltag erlebt und gewertet?

Dazu wurden im Sommer 2013 Experteninterviews in Form offener Gespräche geführt und durch standardisierte quantitative Fragebögen ergänzt (vgl. zur Methode allgemein: Lamnek 1995a/b; Mayer 2004). Jene Teilnehmer, die ihrer namentlichen Nennung zustimmten, sind in Abbildung 1 in der von ihnen gewünschten Form aufgeführt. Insgesamt nahmen 14 Personen an der Untersuchung teil. Insofern, als insgesamt 18 Personen angefragt wurden, kann ein großes Interesse an der Thematik konstatiert werden. Einige Personen haben nur am quantitativen Fragebogen teilgenommen, andere ausschließlich am Experteninterview, einige an beidem. In Abbildung 1 wird vereinbarungsgemäß nicht ersichtlich gemacht, wer an welchen Interviewformen Teil genommen hat. Freundlicherweise gestatteten alle Experten den Mitschnitt der Gespräche. Die Interviews dauerten zwischen dreißig Minuten und zwei Stunden. Den Interviewpartnern wurden zufällige Nummerierungen (IP 1-10) zugeordnet, die ausdrücklich nicht(!) der Reihenfolge entsprechen, in der sie hier aufgelistet sind. Dabei ist es möglich, dass in Abbildung 1 nicht alle Gesprächspartner aufgeführt sind, hingegen einige oder alle, die lediglich an der quantitativen Befragung teilnahmen. Wörtliche Zitate sind anonymisiert, werden aber unterscheidbar bestimmten Gesprächspartnern (z.B. IP X) zugeordnet. Sinngemäße Zitate, die von mehreren Interviewpartnern artikuliert wurden, sind entsprechend gekennzeichnet. 
Name

Jaan Albrecht

Dr. Andreas Bierwirth

Thomas Bößer

Dr. Wolfgang Henle

Christian Korherr

Dirk Kröger

Wolfgang Mayrhuber

Dr. Dieter Reisinger

Jürgen Rother

Ralph Striewski

Dr. Jan Vespermann

Oliver Will

\section{Position}

Chief Executive Officer, Austrian Airlines

ehemaliger Chief Executive Officer, Austrian Airlines

Fleetcaptain, Lufthansa

Accountable Manager

Managing Director Simulator Center Vienna, Berlin, Lufthansa

Flight Training, ehemaliger Flottenchef und Postholder Training, Austrian Airlines

Geschäftsbereichsleiter Flugschulen, Lufthansa Flight Training

Aufsichtsratsvorsitzender Lufthansa

Safety Officer, Austrian Airlines

ehemaliger Leiter der Pilotenselektion, Lufthansa

Safety Officer, Lufthansa

Manager Konzernstrategie

ehemaliger Sprecher Lufthansa Consulting, ehemaliger Sprecher Vereinigung Cockpit, Inhaber einer Flugschule

\section{Abbildung 1: Teilnehmer an der Studie (Quelle: eigene Darstellung)}

Mit Hilfe eines Gesprächsleitfadens (Abbildung 2) wurde versucht, sich während der Interviews stets an der Erkenntnisfrage zu orientieren - gleichwohl wurde aber auch Freiraum für prima vista abweichende, doch als relevant erscheinende Einlassungen gegeben. In den Interviews interessierten weniger einzelne Unternehmen mit ihren Spezifika, als vielmehr die Luftfahrtbranche insgesamt.

Mittels der quantitativen Fragebögen (Abbildung 3 im Anhang; siehe Abschnitt 7.2) sollte ermittelt werden, wie konkrete und identische Fragestellungen an unterschiedlichen Stellen im Unternehmen jeweils bewertet werden. Der Fokus liegt hier entsprechend nicht auf statistischer Aussagekraft, sondern auf der Frage, ob und bei welchen Themen erkennbar in-/homogene Bewertungen vorkommen. Das Herausarbeiten der Denkmuster beschränkt sich auf klar artikulierte Aussagen (vgl. Mayer 2004: 46f.). Durch die Möglichkeit zum beiderseitigen Nachfragen wurde bewusst darauf abgezielt, Missverständnisse auszuräumen.

Das Ziel der Repräsentativität verfolgt die Studie nicht - den strengen methodischen Anforderungen hierfür entspricht sie von daher ebenfalls nicht. Vielmehr geht es um die Bestimmung und Verteilung von Denkweisen, die im informellen, praktischen Alltag vorkommen. Die Befragten wurden gezielt ausgesucht, auch damit nicht nur Mehrheitsmeinungen vertreten sind. Alle Teilnehmer sind aus Fluggesellschaften des deutschsprachigen Raums. Unter den Gesprächspartnern sind Führungskräfte aus dem obersten Management wie auch aus der Linie, beschäftigt sowohl in Größtunternehmen mit über 5.000 Mitarbeitern, als auch in Kleinunternehmen mit unter 50 Mitarbeitern (vgl. auch ICAO 2013). Insgesamt 
ist also zu betonen, dass eine statistische Validität der Aussagen nicht beansprucht wird und der Charakter der Untersuchung vielmehr rein explorativ ist.

\section{Interviewleitfragen}

- Flugsicherheit soll ganzheitlicher, systemischer betrachtet werden. Mit dem International Civil Aviation Organization (ICAO) Document (DOC) 9859 werden nun Luftfahrtunternehmen in die Pflicht genommen, sich Ihr eigenes Safety Management System (SMS) aufzubauen. Hierfür soll ein individueller, in Zusammenarbeit mit der nationalen Luftfahrtbehörde vereinbarter, Acceptable Level of Safety Performance (ALoSP) bestimmt und eingehalten werden.

- Beeinflusst diese Neugestaltung der Flugsicherheitsaufsicht, Ihrer Einschätzung nach, die Gewährleistung hoher Flugsicherheit?

- Würden Sie also eher sagen, hohe Flugsicherheit - die auch über das gesetzliche Mindestmaß hinausgehen kann - und wirtschaftlicher Erfolg spornen sich gegenseitig an, oder behindern sie einander?

- Wie bewerten Sie das?

- Wird Flugsicherheit als Mittel im Wettbewerb verwendet? Wie und woran sieht man das? War es früher anders?

- Würde eine Airline vom Markt belohnt oder bestraft werden, wenn sie Flugsicherheit unabhängig von den Kosten, also mit einem immens großen Budget verfolgt? Hat sich dies im Laufe der Zeit geändert?

- Gilt in Luftfahrtunternehmen die Maxime »Safety First «? Woran zeigt sich das?

- Im Vergleich zu früher, wie weit sind Fluggesellschaften heutzutage von den gesetzlichen Mindestanforderungen in Bezug auf Flugsicherheit entfernt? Warum?

- Gibt es oder gab es Sachzwänge, die sich erschwerend auf Flugsicherheit auswirken? Welche?

- Gibt es in alltäglichen Entscheidungen einen Maßstab für Flugsicherheit, oder geht es bei Flugsicherheit eher darum, kritisch zu evaluieren, ob für eine bestimmte Praxis ein begründbarer Anspruch besteht? Haben Sie dafür Beispiele?

- Wann geht in alltäglichen Entscheidungssituationen Flugsicherheit zu Lasten von Rentabilität und wann nicht?

- Wie wird mit kritischer Loyalität in Luftfahrtunternehmen umgegangen? Haben Sie Beispiele? War das mal anders?

- Welches Schlüsselproblem der Branche hat den größten negativen Einfluss auf Flugsicherheit?

- Gibt es Ihrer Einschätzung nach alltägliche Situationen, in denen Druck auf Beteiligte herrscht, sich im Zweifelsfall für die kostengünstigere und gegen die sicherere Variante zu entscheiden? Oder andersherum? Hat sich das im Laufe der Zeit verändert?

- Wie wird sich Ihrer Einschätzung nach, die Flugsicherheit in Zukunft entwickeln? Warum?

Abbildung 2: Gesprächsleitfaden (Quelle: eigene Darstellung) 


\section{Ergebnisse der Studie: Formale Praxis}

\subsection{Am Beispiel der Öffentlichkeitsarbeit von Luftfahrtunternehmen}

Im Ersten interessierte uns die formale Öffentlichkeitsarbeit relevanter Luftfahrtunternehmen, die prägend für Geschäftsberichte, Internetauftritte und sonstige Publikationen einzelner Unternehmen ist. Derlei Verlautbarungen wurden im Hinblick auf die Aspekte Unternehmenserfolg und Flugsicherheit auf dem Wege einer Dokumentenanalyse zusammengetragen, was - entlang ausgewählter Statements insgesamt zu folgenden Ergebnissen führt.

\subsubsection{Interpretation und Relevanz des Unternehmenserfolges}

Grundsätzlich zeigen sich bei allen Unternehmen große Ähnlichkeiten in der inhaltlichen Interpretation und in der wahrgenommenen Relevanz des Unternehmenserfolgs - dies auch unabhängig davon, ob das betrachtete Unternehmen gerade Rekordgewinne erzielt oder überhaupt erst Gewinne erzielen möchte. Aus - Wir haben die stärkste Bilanz` wird gefolgert, man müsse den Profit noch mehr erhöhen (vgl. Lufthansa 2013: 3; vgl. EasyJet 2013: 6; Ryanair 2013: 46). Dies ist aber nur möglich, wenn auch an den für das Unternehmen gestaltbaren Aspekten, insbesondere den betriebsinternen Kosten, angesetzt wird (vgl. Air Berlin 2013; British Airways 2013: 3; Lufthansa 2013: 3; Ryanair 2013: 4). Es gilt sicherzustellen, dass » returns are maximised « (EasyJet 2013: 6). Um die für einen Wettbewerbsvorteil erforderlichen niedrig(st)en Kosten zu ermöglichen, muss freier Wettbewerb gesichert werden. Nur dann kann überschüssige Liquidität an die Aktionäre zurückgegeben werden (vgl. EasyJet 2013: 16; Ryanair 2013: 7). »Wir werden alles tun, unsere [Kostensenkungs-] Ziele zu erreichen (...) [um] unsere Position als Weltmarktführer auszubauen« (Lufthansa 2013: 6f.).

Korrespondierend damit soll eine »wertorientierte Steuerung in sämtlichen Entscheidungsprozessen [verankert werden] " (Lufthansa 2013: 15). Hierfür wäre dann auch die Unternehmenskultur zu ändern (vgl. Air Berlin 2013: 3; Lufthansa 2013: 95ff.). Die Auswahl der Führungskräfte muss sich am übergeordneten Ziel der Steigerung des Unternehmenswertes ausrichten; als bestgeeigneter Führungsstil erscheint die transformationale Führung (vgl. EasyJet 2013: 34; Lufthansa 2013: 96). Das Unternehmen ist gesamthaft als >Portfolio auszugestalten (Lufthansa 2013: 35). Man muss stets besser sein als die Anderen. Zuletzt werden Kapitalrenditen angestrebt, die in der Luftfahrtbranche bis dato eher unerreichbar erschienen (vgl. Pompl 2007: 8; 121). Der hierfür erforderliche Wandel wird als "schmerzhaft, aber unvermeidlich " (Air Berlin 2013: 6) charakterisiert. In summa muss die Produktivität ständig erhöht werden, ganz gleich wie hoch sie bereits ist (vgl. Ryanair 2013: 57).

\subsubsection{Interpretation und Relevanz von Unternehmensethik im Allgemeinen und Flugsicherheit im Besonderen}

Im Kontext eines verantwortungsvollen Handelns wird v.a. auf wohltätige Zwecke außerhalb des Geschäftsalltags oder Ethik-Ratings hingewiesen. Aber auch 
erschwinglichere Preise und mehr Streckenverbindungen werden als Werte benannt, die den Menschen zu Gute kommen. Firmeninterne Veränderungen werden in der unternehmensethischen Selbstdarstellung nicht problematisiert, auch dann nicht, wenn profitable Standorte geschlossen werden, um anderenorts noch höhere Rentabilität zu erzielen (vgl. EasyJet 2013: 6). Maßnahmen, die den Treibstoffverbrauch senken, gelten als verantwortungsvolles Handeln und sparen zugleich Kosten (vgl. Air Berlin 2013: 52; Lufthansa 2013: 100ff.). Verantwortungsbewusstes unternehmerisches Handeln zeigt sich an allen internen Kostensenkungen, mit denen man dem harten Konkurrenzkampf entgegentritt (vgl. Lufthansa 2013: 100ff.). Verantwortung wird in aller Regel auch als Instrument für wirtschaftlichen Erfolg beschrieben (vgl. EasyJet 2013: 31ff.).

Beispielhaft hierfür ist der Code of Business Conduct and Ethics von Ryanair (2012), der anmahnt, sich an Gesetze zu halten. Denn es gehe darum, einen vernünftigen Return on Investment zu erwirtschaften, der nicht durch Strafzahlungen infolge Gesetzesverstößen gefährdet werden sollte. Schließlich wird auch das Leitprinzip 'Safety First bzw. das Ziel der Flugsicherheit an ein finanzielles Kalkül geknüpft. Schließlich können Zwischenfälle die öffentliche Meinung über das Luftfahrtunternehmen und damit auch seine finanzielle Basis negativ beeinträchtigen (vgl. Air Berlin 2013: 65; British Airways 2013: 9; EasyJet 2013: 26; Lufthansa 2013: 111f.; Ryanair 2013: 50ff.). Das Management hat hier wie ein Aktionär zu denken und zu handeln (vgl. Ryanair 2013: 7). Bezugnehmend auf Flugsicherheit führen gesetzliche Anforderungen zu Kosten. In Bezug auf staatliche Regulierung der Wirtschaft verzerren sie den Wettbewerb und stehen Kostensenkungen im Weg, so dass Luftfahrtunternehmen die Gesetzgebung entsprechend ihrer Ziele beeinflussen müssen. Regeln muss man gut kennen, um keine kostspieligen Strafen zu erleiden (vgl. EasyJet 2013: 30).

Wie die aufgeführten Statements zeigen, sind einschlägige Verlautbarungen relevanter Fluggesellschaften durch eine starke Fixierung auf fortgesetzte Kostensenkungen und hiermit verbundene Renditesteigerungen geprägt. Unternehmensethische Verhaltensweisen - und hier insbesondere eine hohe Flugsicherheit - werden dabei allerdings als weithin kompatibel mit diesen Bestrebungen gewertet, womit unausgesprochen, aber dennoch recht deutlich, der unternehmensethischen Harmoniethese gefolgt wird. Überlegungen oder konkrete Beispiele, die der Konfliktthese der Unternehmensethik entsprechen würden, bleiben weitestgehend ausgespart.

\subsection{Am Beispiel eines gesetzgebenden Prozesses}

Die Relevanz der unternehmensethischen Konfliktthese zeigte sich hingegen exemplarisch im Kontext einer Debatte im Europäischen Parlament, die sich auf eine Neuregelung der Flugdienstzeiten bezog und bei der unterschiedliche Anspruchsgruppen zu Wort kamen (vgl. Europäisches Parlament 2013):

Vertreter der Fluggesellschaften plädierten hier für großzügigere gesetzliche Rahmenbedingungen, um Personal wirtschaftlicher einsetzen zu können. In der Praxis sollte das sog. Fatigue Risk Management (FRM) nichtsdestotrotz auch 
Sicherheit gewährleisten - dies sogar für den Fall, dass die gesetzlichen Regelungen über die von der Luftfahrtmedizin empfohlenen Maximalzeiten hinausgehen. Arbeitnehmervertreter warnten dagegen, dass Luftfahrtunternehmen dieses Risiko-Management ganz im Gegenteil aus rein wirtschaftlichen Gründen auf Kosten der Sicherheit ausnutzen würden. Diese Einschätzung teilten auch einige Parlamentarier.

Luftfahrtbehörden wiesen auf die notwendige Harmonisierung von Regelungen innerhalb Europas ebenso hin wie auf die Eigenverantwortung der Luftfahrtunternehmen. Es wurde versichert, dass die entsprechenden Anspruchsgruppen bei der Erstellung des Gesetzesvorschlags einbezogen werden. Einige Parlamentarier bemängelten jedoch das Fehlen einer dezidiert an der Sicherheit ausgerichteten Argumentation, die erklärt, weshalb die European Aviation Safety Agency (EASA) es riskiert, das von der Medizin empfohlene Maß zu überschreiten. Abschließend mahnte der Vorsitzende an, sich daran zu erinnern, wie aufwändig es war, diesen einheitlichen Entwurf überhaupt erarbeitet zu haben.

Insgesamt zeigt sich damit, dass in (politischen) Debatten, innerhalb derer verschiedene Anspruchsgruppen zu Wort kommen, eher Argumentationen im Sinne der unternehmensethischen Konfliktthese festzustellen sind, wohingegen öffentliche Verlautbarungen, die von Seiten einzelner Unternehmen getätigt werden, eher der unternehmensethischen Harmoniethese folgen. Dies deutet nicht zuletzt darauf hin, dass dialogische Prozesse durchaus andere Ergebnisse hinsichtlich des ethisch Richtigen zeitigen als quasi monologische Verfahren (vgl. dazu grundlegend: Steinmann/Löhr 1991; Ulrich 2016).

\section{Ergebnisse der Studie: Informelle Praxis}

\subsection{Denkmuster zwischen Harmonie und Konflikt}

In den Interviews wurde prima vista eine eher harmonische Sichtweise vertreten: Flugsicherheit, so das allgemeine Credo, kurbelt langfristig den wirtschaftlichen Erfolg an, zahlt sich finanziell somit letztlich aus. Eine in dieser Form durchgängige Argumentation war allerdings eher die Ausnahme. Vielmehr gingen die harmonisch geprägten Aussagen häufig mit konfliktorientierten Aussagen einher. Auch wurden zunächst eindeutige Aussagen im Sinne der Harmoniethese (größerer wirtschaftlicher Erfolg infolge höherer Flugsicherheit) zuweilen im Laufe des Gesprächs kritischer eingeschätzt und relativiert.

Auffällig war, dass auch äußerst unterschiedliche Einschätzungen vorkamen. So etwa die Einschätzung, dass Flugsicherheit und wirtschaftlicher Erfolg von einander "abgekoppelt " (IP 1; 7) seien. Wenn man sich an die Gesetze halte, sei es "unmöglich unsicher zu sein «(ebd.), so dass Flugsicherheit einen "No-Factor " (ebd.) darstelle, den man »in diesem Sinne « (ebd.) nicht beachten muss, weil man sich beim Kunden damit nicht differenzieren kann, »und mache einfach, was [gesetzlich] vorgeschrieben ist " (ebd.). Aber auch umgekehrt wurde dezidiert behauptet, »legal heißt noch lange nicht sicher « (IP 2). Ein gleichzeitiges Höchst$\mathrm{maß}$ an Flugsicherheit und wirtschaftlichem Erfolg wurde teils für möglich, teils 
aber auch für unmöglich erachtet. Vorwiegend war allerdings die Einschätzung, man müsse Flugsicherheit mit wirtschaftlichem Erfolg ausbalancieren.

Übereinstimmend wurde der extrem harte Wettbewerb mit »katastrophal niedrigen Margen « (IP 3) und "gnadenloser Preissensibilität « (ebd.) als Last deklariert, weil man somit auch »unmögliche Erwartungen der möglicherweise unkundigen Geldgeber beachten muss « (IP 4). Man könne nur an internen Kosten arbeiten und müsse dies auch tun. "Eine rein ethische Entscheidung kann ich mir in unserer Branche nicht vorstellen " (IP 3). Oder aus anderer Perspektive: «Flugsicherheit ist ein schlechtes Beispiel für ethisches Handeln. Da handle ich nicht unethisch, sondern doof, da gefährde ich mich selber, wenn ich glaube, Gesetze brechen zu müssen« (IP 1).

Letztendlich wird die Bestimmung eines quantitativen Zielwertes für Flugsicherheit als wirtschaftliche Notwendigkeit begründet. Denn Zwischenfälle mindern das Vertrauen der Kunden und gefährden damit das wirtschaftliche Überleben des Unternehmens. Aber auch aus Zwischenfällen resultierende Kosten haben einen negativen finanziellen Effekt: "If you think safety is expensive, try an accident" (IP 2). So müsse es eine »balance of powers « (IP $2 ; 3 ; 5 ; 6 ; 7$ ) zwischen den Ansprüchen Flugsicherheit und Wirtschaftlichkeit innerhalb eines Unternehmens geben, damit »der eine den anderen nicht killen darf « (IP 6). Aber auch hier wird nicht immer ein Konflikt erkannt, da reibungslose Vorgänge wirtschaftlichen Prozessen entsprächen und damit die Voraussetzung für hohe Sicherheit seien (vgl. IP 10). Genau damit wurde jedoch von einem nicht unerheblichen Teil der Befragtengruppe ein herrschender Druck im Alltag begründet: Dieser wird darin gesehen, einen Betrieb, der z.B. durch technische Gebrechen oder schlechtes Wetter ins Stocken gekommen ist, trotzdem weiter am Laufen zu halten, auch wenn es dem persönlichen Verständnis von Sicherheit zuwider läuft. In solch einer Situation greift jedoch - so ein Befragter, der diese Einschätzung nicht teilt - ein in der Branche vorhandenes Ethos, dass »Profis « (IP 7) wissen, wie weit man gehen kann.

Bedauernswert sei jedoch, so die einhellige Meinung, dass der Passagier so gut wie nicht einschätzen kann, was eine Fluggesellschaft für Flugsicherheit tut. Er bemerkt nicht, ob eine Situation sehr knapp vor einem Zwischenfall stand und nur gerade noch einmal gut gegangen ist. Für ein Luftfahrtunternehmen ist es zwar essentiell, sich an den Bedürfnissen des aktuellen "Zentralkunden " (IP 1) auszurichten, jedoch geht es dabei in der Regel eher um das Essen und die Filmauswahl. Die Konsequenzen einer Reduzierung des Sitzabstandes z.B. kann schnell und direkt an finanziellen Kennzahlen abgelesen werden; eine Reduzierung von Schulungsaufwand ebenfalls - und zwar an den sofort eingesparten Kosten. Umso schwieriger zu messen aber ist der Einfluss auf den Sicherheitslevel. Dieser kann sich, wenn überhaupt, erst nach Jahren bemerkbar machen und wird selbst dann (zumindest noch) nicht eindeutig auf die ursprüngliche Reduzierung des Schulungsaufwandes zurückgeführt werden können. Eine solche Argumentationslinie offenbarte sich in den meisten Interviews. Und vielleicht sind die Verantwortlichen für diese Entscheidungen dann schon längst in einem anderen Unternehmen - womöglich in einer anderen Branche (vgl. IP 5). 
Kunden machen ihre Kaufentscheidung kaum mehr von dem guten Ruf abhängig, den eine bestimmte Airline in puncto Sicherheit genießt. Und falls doch, sind dies zumeist "nur die Niedrigzahler, die wenig fliegen und leicht substituierbar sind « (IP 8). Trotzdem wurde von nicht wenigen auch hervorgehoben, dass Sicherheit ein Teil des Markenauftrittes ist: gelegentlich wurde sogar zuversichtlich die Möglichkeit beschrieben, wie man Kunden überzeugen könne, für mehr Sicherheit auch höhere Ticketpreise zu zahlen (vgl. IP 5; 10). Es sei aber ganz natürlich, dass Fluggesellschaften auszuloten versuchen, wie wenig Sicherheit noch ausreicht - mit unterschiedlichen Maßstäben. Zunehmend muss man sich auch mit solchen Fragen auseinandersetzen, besonders wenn die Konkurrenz dies schon getan hat und bisher »nichts passiert " (IP 9) ist. Solch ein Ausloten sei mit neuartigen Überwachungssystemen nun auch »neutraler bewertbar (IP 8).

Durchaus unterschiedlich wurden gewisse »Stellschrauben« (IP 5; 6) mit ihren Auswirkungen auf das Sicherheitsniveau und/oder den wirtschaftlichen Erfolg bewertet. Somit kann »ein persönliches Verständnis von Sicherheit (...), dass so wenig wie möglich passiert " (IP 6) an anderer Stelle auch als "päpstlicher als der Papst « (IP 8) bewertet werden, so dass derjenige dann auch »schnell mal raus rotiert wird « (ebd.). Der »Ehrenkodex (...) [ist] schon sehr in Frage gestellt (...) Gott sei Dank haben wir es mit so [regulatorischen] Peinlichkeiten zu tun (...,) die die Stange [in Bezug auf Flugsicherheit] hoch halten. Früher haben es die Menschen getan« (IP 5).

Fast durchgehend wurde der Sicherheitsstandard in der Luftfahrtbranche mit Aussagen im Sinne von "Die Bandbreite ist sehr (IP 10)/dramatisch (IP 6)/erschreckend (IP 3) groß « umschrieben. Der Anspruch hoher Sicherheit steht nicht mehr unangefochten an erster Stelle. Dies sei aber auch gar nicht nötig, so nicht wenige Einschätzungen, da die regulatorischen Anforderungen so strikt geworden sind und ein professionelles Verständnis der Verantwortlichen Sicherheit gewährleistet (vgl. IP 7; 10). In der Praxis ist das Ziel nicht mehr Flugsicherheit zu steigern, sondern Kosten zu senken und dabei möglichst keine Einbußen bei der Sicherheit hinzunehmen (vgl. IP 3; 5). "An dieser Schraube wird momentan so krass gedreht, wie in meiner (...) Karriere noch nie « (IP 5). Man ist dazu übergegangen, sich nur an Vorschriften zu halten "sogar unter Ausnutzung von Gesetzeslücken. (...) Der Level der freiwilligen Sicherheitssicherstellung bei den Airlines ist erschreckend gegen Null gesunken « (ebd.). »Die Ethik (...) ist heute absolut tabu, weil sie Kosten verursacht « (IP 5; vgl. auch IP 3). »Dieser Stellschraube ist praktisch keine Gegenkraft entgegengesetzt « (IP 5). Stellenbesetzungen haben sich »viel extremer zu einem betriebswirtschaftlichen Ellenbogengerangel entwickelt. (...) Es sind heute andere Werte (ebd.). Heute sind die einzelnen Abteilungen »Unterabteilungen einer Gewinnerzielungsabsichtsmaschine (ebd.). In Bezug auf Sicherheit, so ein Befragter, ruht man sich heute auf Lorbeeren aus, die in vielen Jahrzehnten erarbeitet wurden (vgl. ebd.). Die entgegengesetzte Sichtweise betont, dass Kostensenkungen jedoch die Flugsicherheit gar nicht betreffen, da sie intelligent umgesetzt werden - eben nur da, wo sie die Flugsicherheit nicht beeinflussen oder sogar fördern (vgl. IP 8; 10). Zudem seien etwa »fliegerische Fertigkeiten der 
Piloten durch Technik gecovert« (IP 8). Diese Einschätzung ist jedoch ebenfalls sehr kontrovers.

»Das richtige Maß [an Flugsicherheit] zu finden, wird immer eine Diskussion sein « (IP 6). Besonders in Einzelfällen ist es häufig eine »Bauchentscheidung « (IP 9). »Mir würde gefallen [Flugsicherheit an der Frage zu messen], was tun Unternehmen für Flugsicherheit, und nicht [am] Ergebnis, das von so vielen nicht beeinflussbaren Faktoren bestimmt wird " (IP 6). Nach einem solchen Motto in der Luftfahrtbranche handeln zu können, erscheint jedoch zusehends unwahrscheinlicher. Analytische und beweisbare Zusammenhänge zu quantitativen Zielwerten werden eingefordert, während vergleichsweise geringe wirtschaftliche Krisen mit immer härteren Bandagen ausgefochten werden.

Zusammenfassend vertraut man dennoch eher auf weiter steigende oder zumindest nicht sinkende Flugsicherheitsstandards - des technologischen Fortschritts wegen und aufgrund der Einschätzung, dass der Markt schlechtere Flugsicherheit sanktionieren wird. Schlussendlich sei Sicherheit notwendige Voraussetzung für das wirtschaftliche Überleben des Unternehmens. Eine Verschiebung der Verantwortung hin zu den Unternehmen würde keinen großen Unterschied machen, da die Behörden, vornehmlich die europäischen, auch bisher schon überfordert waren.

Interessant in diesem Zusammenhang war auch die häufig geäußerte Einschätzung, dass mit der Neuregelung des SMS große Fluggesellschaften gegenüber Kleineren (finanziell) benachteiligt werden. Große Firmen müssen nämlich wegen der höheren Anzahl von Flugbewegungen mehr Aufwand für Flugsicherheit betreiben, um absolut gesehen auf eine gleiche Anzahl von Vorfällen zu kommen wie kleinere Unternehmen. In statistischer Wahrscheinlichkeit gerechnet - wie es in manchen Luftfahrtunternehmen Praxis ist - muss das Sicherheitsziel des Unternehmens, und damit eines jeden Fluges, letztendlich anspruchsvoller sein, als bei einer kleineren Fluglinie. Denn: Bei weniger Flugbewegungen kann man gewissermaßen auf die Statistik hoffen. Schließlich sind absolute Zahlen und ob man »in der Zeitung steht « (IP 9) nun mal das, was die Öffentlichkeit interessiert. Aus dieser Perspektive erscheint auch ein guter Ruf, ein hoher Markenwert, als Constraint, also als gewisser Anreiz für Sicherheit - oder aber dafür, diesen finanziellen Wert nicht mit Einsparungen, die sich auf die Sicherheit auswirken, aufs Spiel zu setzen. Hohe Flugsicherheit zu verfolgen, kostet einfach, das sei ganz klar. So ist es auch schon vorgekommen, dass Fluggesellschaften nach einem großen Vorfall Bankrott gegangen sind, jedoch unter anderem Namen - tatsächlich aber die gleiche Firma verkörpernd - neu gegründet wurden. Ein geringerer Markenwert oder ein kleinerer Flugbetrieb kann aus dieser Blickrichtung als geringerer Anreiz für Flugsicherheit interpretiert werden; oder eben als größerer Anreiz, "kommerziell zu optimieren (...), an vertretbare Grenzen zu gehen « (IP 8).

Mit Blick auf unsere Fragestellung zeigt sich damit, dass die Anschauungen zwischen einzelnen Befragten, häufig aber auch innerhalb einer einzelnen Person, stark oszillieren. Es schlagen, wenn man so sagen darf, >beide Seelen in der Brust der Experten. So koexistiert die Überzeugung, dass der Markt für optimale Flugsicherheit sorgt, mehr oder minder friedlich mit dem Bewusstsein, dass der Kunde 
weit davon entfernt ist, Flugsicherheitslevels konkurrierender Luftfahrtunternehmen auch nur annähernd objektiv einschätzen zu können. In gewisser Weise scheint es damit so zu sein, dass die abstrakte Frage "Ist Ethik ein Kosten- oder ein Erfolgsfaktor? «, die in der Unternehmensethik-Debatte zwar gestellt, aber noch nicht wirklich beantwortet wurde, sich für Luftfahrtunternehmen konkretisiert in der Frage »Ist Flugsicherheit ein Kosten- oder ein Erfolgsfaktor? « - wobei diese konkrete Frage nicht nur nicht beantwortet, sondern innerhalb der Unternehmen noch nicht einmal offen gestellt wurde.

\subsection{Bewertungen zum Verhältnis von wirtschaftlichem Erfolg und Flugsicherheit}

In Ergänzung der Experteninterviews wurde ein Fragebogen konzipiert, der das Verhältnis zwischen wirtschaftlichem Erfolg und Flugsicherheit mittels spezifischer Fragestellungen nochmals auf quantitative Weise ausleuchten sollte und der von maximal 9 Personen ausgefüllt wurde. Entsprechend dieser geringen Teilnehmerzahl erheben die in Abbildung 3 wiedergegebenen Befragungsergebnisse selbstredend nicht den Anspruch auf Repräsentativität, sondern verstehen sich rein explorativ. Es geht grundsätzlich um die Frage, ob hohe Flugsicherheit und wirtschaftlicher Erfolg einander anspornen, oder einander im Weg stehen. Dazu sollten die Teilnehmer die Fragen jeweils mit einer Zahl zwischen 1 und 5 beantworten. Wobei 1 'Stimme voll zu<, 2 >Stimme eher zu<, 3 >teils-teils<, 4 >Stimme eher nicht zu< und 5 >Stimme gar nicht zu<, bedeutet.

Als besonders bemerkenswertes Ergebnis dieser quantitativen Befragung erscheint die hohe Zustimmung (1,2) zu der These, dass Flugsicherheit sich langfristig auszahlen wird (Harmoniethese), in Verbindung mit der exakt gleich hohen Zustimmung $(1,2)$ zur - quasi entgegengesetzten - (Konflikt-)These, dass Flugsicherheit feste internationale Standards braucht, damit nicht auf Kosten der Flugsicherheit Wettbewerb betrieben wird (vgl. sinngemäß auch Frage 3). Die weitgehende Unterschiedenheit zwischen einem Verständnis der Flugsicherheit als Kosten- oder als Erfolgsfaktor, die im Rahmen der Interviews ja bereits konstatiert wurde, wird in der Befragung mithin quasi bruchlos fortgeschrieben. Interessant erscheint ferner auch die deutliche Ablehnung $(3,6)$ der These, dass Renditesteigerung die schlussendliche Maßgabe eines Unternehmens sein sollte. ${ }^{2}$ Diese normative Ablehnung steht in erkennbarem Widerspruch zu den formalen Verlautbarungen der Luftfahrtunternehmen (vgl. Abschnitt 6.1) wie auch zu den persönlichen Wahrnehmungen der befragten Experten (vgl. Abschnitt 7.1), die einen steigenden Druck zur fortgesetzten Renditesteigerung ja objektiv feststellen. Die Fragen 8, 9 und 12 zielen auf das Konfliktbewusstsein zwischen Flugsicherheit und Unternehmenserfolg im konkreten Arbeitsalltag ab. Die Antwortwerte bewegen sich sehr nah am Mittelwert (Antwortmöglichkeit steils-teils<). Zu beachten ist hier jedoch die jeweils hohe Standardabweichung $(2,7 ; 3,61 ; 2,94)$. Bei diesen Fragen schwindet die breite Zustimmung zur Harmoniethese (vgl. Frage 1) bzw. zur Konfliktthese (vgl. Frage 13) zu Gunsten eines differenzierten Bildes. Ein Teil der Befrag-

2 Sinngemäß kann auch die hohe Zustimmung $(1,6)$ zu Frage 14 interpretiert werden. 
tengruppe sieht hier jeweils weiterhin eine harmonische Koexistenz von Flugsicherheit und Erfolg, der andere hingegen erkennt Konfliktpotenzial. Interessant ist hierbei zu bemerken, dass die Wahrscheinlichkeit, dass der Befragte diese Thesen konfliktbewusst bewertet hat, mit seiner beruflichen Nähe zum Flugbetrieb eher steigt.

Zur Fragestellung des Beitrags zurückkehrend zeichnet die Auswertung der quantitativen Interviews, ähnlich wie die der qualitativen Interviews, ein nebeneinander von Harmonie und Konflikt. Oszillationen der unterschiedlichen Bewertungen zwischen den Befragten, aber auch innerhalb einer einzelnen Person, können nun vielleicht etwas geordnet werden. Abstrakte Thesen zum Verhältnis von wirtschaftlichem Erfolg und Flugsicherheit werden generell recht homogen harmonisch beantwortet. Bewertungen konkreter Fragestellungen hingegen werden differenzierter beurteilt - diejenigen, die tagtäglich mit diesen Fragen zu tun haben, bewerten diese eher konfliktorientiert. Die quantitativen Interviews könnten darauf hinweisen, dass unterschiedliche Bewertungen von Harmonie oder Konflikt sich auch mit dem Aufgabenbereich bzw. der Vertrautheit mit einer konkreten Fragestellung der jeweiligen Person unterscheiden. Deswegen wollen wir nun sehen, wie mit dieser Verschiedenheit umgegangen wird. Dafür kehren wir zu den Experteninterviews zurück.

\subsection{Zum Diskurs über Harmonie oder Konflikt}

Die Ergebnisse der qualitativen und quantitativen Interviews fügen wir nun zusammen, um die Auseinandersetzung mit der Koexistenz von Harmonie- und Konfliktthese innerhalb der Luftfahrtunternehmen besser nachzuvollziehen.

Dass die Unternehmensführung auch sicherheitsrelevante Informationen erhält, zeige sich an den unbedingt notwendigen und gut funktionierenden »Feedbackschleifen « (IP 8; vgl. IP 1; 10) aus der Praxis, wie sie auch im SMS gefordert werden. Gemeinsam mit quantitativen Methoden kann so ganz analytisch die »root cause (IP 10) eliminiert und Flugsicherheit gesteigert werden. "Intelligente Manager freuen sich über gute Argumente (IP 2). Denn "auch sie stehen unter großem Druck « (ebd.) und »der Vorstand muss sich vor den Fondsmanagern verantworten « (ebd.). Leitende Angestellte im Flugbetrieb würden dem Top Management durchaus sagen »wenn was im Argen ist « (IP 8). Inwiefern diese Rückmeldungen jedoch kritisch loyal sein können, wurde von einigen in Frage gestellt, da man diese leitenden Positionen bei fehlendem »Management-Verständnis « (IP 8) nicht lange behält bzw. erst gar nicht bekommt. Dabei wird dieses `ManagementVerständnis` von denen definiert, die dann über die Besetzung entscheiden.

»Wenn man Incident Reports liest, ist das eine andere Mentalität, als wenn man sich mit Wirtschaftszahlen (...) beschäftigt. (...) Das verstehen die nicht« (IP 2). Im oberen Management und in Organisationsbereichen, die wenig mit dem fliegerischen Alltag zu tun haben, wurde das Mittel-Zweck-Verhältnis in Luftfahrtunternehmen mitunter sehr harmonisch bewertet. Das System würde sich von selbst optimieren und die Schlechteren automatisch von den Besseren lernen (vgl. IP 10). Befragte, die hingegen am Flugbetrieb selbst beteiligt sind, bewerteten dies teils 
äußerst kritisch. Insbesondere konkrete Fragestellungen wurden von der >bürokratischen und der 'praktischen Teilgruppe sehr unterschiedlich bewertet, innerhalb der jeweiligen Gruppe hingegen ziemlich homogen (vgl. Abschnitt 7.2). Diesbezüglich wurden Beteuerungen, für ein Höchstmaß an Sicherheit einzustehen, gelegentlich als leere Lippenbekenntnisse bewertet, die gegenüber dem wirtschaftlichen Druck chancenlos seien (vgl. IP 2; 5).

Eine »Kopplung von Managergehältern an einen nachweisbaren Flugsicherheitsstandard einer Airline« (IP 2) sei deswegen wünschenswert. Oder auch »eine Institution zu installieren, die schonungslos aufdeckt über Flugsicherheitsdefizite " (IP 5). Außerdem braucht Flugsicherheit feste internationale Standards. Diese Bewertung war wieder recht homogen in der gesamten Befragtengruppe vertreten (vgl. Abbildung 3). »Ich glaube grundsätzlich, dass jede gesetzliche Forderung, die unklar formuliert ist, die gesamte Bandbreite des Handelns zulässt« (IP 2). In Bezug auf die Neugestaltung der Flugsicherheitsaufsicht wurde vermerkt: »Behörden überlassen es den Unternehmungen vollkommen und schauen es sich mal an " (IP 6). Doch auch hier gab es eine abweichende Bewertung: Es gibt »keinen Spielraum « (IP 1), den die Airlines in Bezug auf ihren Sicherheitslevel umsetzen können.

Resümieren wir die Ergebnisse der qualitativen und quantitativen Interviews zur informellen Praxis, so zeichnet sich ein Umfeld ab, in dem der harte Wettbewerb eine Ausrichtung an den Kunden fordert - Kunden, denen kaum zugemutet wird, dass sie den Sicherheitsstandard eines Luftfahrtunternehmens bewerten können. Einerseits wird auf ein persönliches Ethos und analytische Methoden innerhalb des Unternehmens in Bezug auf Flugsicherheit vertraut, andererseits wird ein fehlendes Verständnis über Sicherheit bemängelt und ein persönliches Ethos als zunehmend schwerer zur Geltung zu bringen beurteilt. Es scheint, wie wenn es auf die Frage, ob Flugsicherheit ein Kosten- oder Erfolgsfaktor ist, durchaus unterschiedliche Antworten gäbe, sich jedoch einige davon kaum Gehör verschaffen können oder nicht ernst genommen werden.

Entsprechend der geringen Teilnehmerzahl dieser Studie sind die Ergebnisse selbstredend weit davon entfernt, abschließend zu sein. Sie zeigen jedoch auf, dass gegenüber Verlautbarungen, die eine durchgängige Harmonie im Verhältnis von wirtschaftlichem Erfolg und Flugsicherheit zu Grunde legen, eine gewisse Skepsis angebracht sein könnte. Umso interessanter wäre weitere Forschungsarbeit in diesem Gebiet.

\section{Diskussion der Ergebnisse und Fazit}

Wie kann gewährleistet werden, dass Flugsicherheit effektiv gesteigert wird (vgl. ICAO 2013: 5-1; Reason 1990)? Unternehmensethisch ausgedrückt: Wie kann verantwortungsvolles Handeln im Sinne hoher oder gar steigender Flugsicherheit als eigenständiger Anspruch sichergestellt werden?

Jemandem, der im harten Wettbewerb steht, ist verantwortungsvolles Handeln nicht ohne Weiteres zuzumuten, wenn dies unternehmerische Nachteile zur Folge 
hat. Das haben Dionne et al. (vgl. 1997) besonders für kleine, in finanzieller Not stehende Luftfahrtunternehmen aufgezeigt. Solche Unternehmen tendieren dazu, an Flugsicherheit zu sparen. Zwar wird ein professionelles Ethos, im Sinne von Safety First, regelmäßig als grundlegend vorausgesetzt, doch wird mindestens genauso häufig darauf hingewiesen, dass der Rechtfertigungsdruck für solch ein Verständnis zugenommen hat bzw. dass diese Art von professioneller Gesinnung stellenweise sogar gänzlich verloren gegangen ist.

Gegenwärtig scheint man dennoch prioritär auf eine (individuelle oder institutionelle) Ethik zu vertrauen, die auch in einem harten Wettbewerb greift: Flugsicherheit soll zunehmend eigenverantwortlich, flexibel und dezentral von Luftfahrtunternehmen gestaltet werden (vgl. ICAO 2013: 2-32; 5-3ff.; Kapitel 2).

Der vorliegende Beitrag ist vor diesem praktischen Hintergrund und eingedenk des unternehmensethischen Diskussionsstandes der Frage nachgegangen, ob Flugsicherheit und wirtschaftlicher Erfolg unweigerlich in einem harmonischen Verhältnis zueinander stehen (Harmoniethese), oder ob diese Zielsetzungen im Zuge weiterer Deregulierungen bei gleichzeitig hohem oder noch weiter steigendem Wettbewerbs- und Erfolgsdruck potenziell in Konflikt geraten können (Konfliktthese). Nachgegangen wurde dieser Frage vermittels verschiedener empirischer Untersuchungen, insbesondere einer Dokumentenanalyse (vgl. Kapitel 6) sowie explorativer Experteninterviews und -befragungen (vgl. Kapitel 7). Im Ergebnis ist deutlich geworden, dass die Luftfahrtunternehmen - wie viele andere Unternehmen anderer Branchen auch - einem kontinuierlich steigenden Wettbewerbs- wie auch Renditedruck unterliegen, weshalb die Relevanz der Zielsetzung >wirtschaftlicher Erfolg für die Verantwortlichen in den Unternehmen weiter zunehmen wird.

Was dies für die Relevanz der (verantwortungsethisch möglicherweise bedeutsameren) Zielsetzung >Flugsicherheit $<$ bedeutet, ist aus Sicht der Verantwortlichen in den Luftfahrtunternehmen keinesfalls eindeutig bestimmt - vermutlich auch noch kaum umfassender bedacht. Unsere Untersuchungen ergaben hier, dass im ersten Zugriff und zuweilen auch darüber hinaus ein harmonisches Verständnis vorherrscht, das implizit auf die ethischen Steuerungsmechanismen des (funktionierenden) Marktes abstellt und kurz gesprochen davon ausgeht, dass im Wettbewerb nicht wirtschaftlich erfolgreich sein kann, wer den (hier: Flugsicherheits-)Interessen und Bedürfnissen seiner Kunden nicht hinreichend entspricht. In zahlreichen Interviews, aber auch in bestimmten Fragestellungen unserer quantitativen Untersuchung, wurde allerdings auch deutlich, dass der Glaube an die harmonische Dependenz relevanter Zielsetzungen von Luftfahrtunternehmen nicht uneingeschränkt und unverbrüchlich ist. Vielmehr besteht eine Konflikthaftigkeit zwischen Erfolg (steigende Rendite) und Ethik (hohe Flugsicherheit), welche im Zuge einer intensiveren gedanklichen Auseinandersetzung mit dieser Problematik durchaus anerkannt und eingeräumt wird. So wurde beispielsweise der marktidealen Überlegung, dass Flugpassagiere natürlich eine hohe Flugsicherheit erwarten und erfolgsorientierte Luftfahrtunternehmen dieser Erwartung deshalb unbedingt nachzukommen haben, die - auf systematisches Marktversagen abstellende - Überlegung entgegengehalten, dass es Flugpassagieren praktisch unmöglich ist, 
die Flugsicherheit eines Luftfahrtunternehmens (ex ante) auch nur ansatzweise zu beurteilen. An diesem Beispiel zeigt sich mit Blick auf die einschlägige Debatte in der Unternehmensethik, dass Unternehmen für ethische Verhaltensweisen nur dann von ihren Anspruchsgruppen sbelohnt`werden können, wenn letztere die Handlungsweisen der Ersteren kompetent beurteilen können. Eben dies ist de facto allerdings häufig gar nicht möglich (vgl. dazu grundlegend: Vogel 2006; Valor 2008; Kuhn/Weibler 2011).

Wenig wahrscheinlich wirkt in diesem Licht, dass die Neuregulierung der Flugsicherheitsaufsicht im Rahmen des International Civil Aviation Organization (ICAO) Document (DOC) 9859 ihr Ziel erreicht (vgl. ICAO 2013: 5-1; Kapitel 2) - nämlich ein grundlegendes und eigenverantwortliches Unternehmensziel effektiver Flugsicherheitssteigerung unter Beachtung konfligierender Ziele innerhalb der Organisation. Dies scheint aufgrund der Ergebnisse unserer Studie aktuell wie zukünftig als eher unwahrscheinlich. Zwar sind Verbesserungen der Sicherheit nicht auszuschließen, doch werden diese kaum das höchst mögliche Maß erreichen. In Folge zunehmender Deregulierung interessiert die Akzeptanz, der Vergleich zu Wettbewerbern, die Benchmark. Kaum jedoch, wo sich der Level absolut befindet.

Erst wenn man Luftfahrtunternehmen die Möglichkeit nimmt (oder einschränkt), ihren Sicherheitslevel selbst zu bestimmen, wird (geringere) Flugsicherheit nicht als Instrument der finanziellen Optimierung missbraucht werden können.

Eine derartige (über)staatliche Regulierung eines (Mindest-)Flugsicherheitslevels etwa wäre jedoch eine konträre Forderung zu den Plänen maßgeblicher Institutionen wie der Europäischen Kommission, der European Aviation Safety Agency (EASA) oder der ICAO (vgl. Commission of the European Communities 2001; Europäisches Parlament 2013; ICAO 2013: 2-32), die sich auf Reasons (1990: 480) Theorie und seine Annahme eines harmonischen Verhältnisses von Flugsicherheit und wirtschaftlichem Erfolg berufen: »in the long term, these (two distinct goals: production and safety) are clearly compatible."

Abschließend möchten wir nochmals auf die begrenzte Datenbasis bei der empirischen Untersuchung verweisen, die natürlich eine Repräsentativität der Ergebnisse ausschließt. Eine endgültige Beantwortung der Forschungsfrage ist damit im Rahmen dieser Studie selbstredend nicht möglich. Der Beitrag ist rein explorativ $\mathrm{zu}$ verstehen, hofft aber weitere notwendige Forschungsarbeit in diesem Themenbereich anzustoßen und hierfür gleichwohl relevante Orientierungen und Anknüpfungspunkte entwickelt zu haben. 


\section{Anhang}

\section{Einleitung Fragebogen}

Flugsicherheit soll ganzheitlicher, systemischer betrachtet werden. Mit dem International Civil Aviation Organization (ICAO) Document (DOC) 9859 werden nun Luftfahrtunternehmen in die Pflicht genommen, sich Ihr eigenes Safety Management System (SMS) aufzubauen. Hierfür soll ein individueller, in Zusammenarbeit mit der nationalen Luftfahrtbehörde vereinbarter, Acceptable Level of Safety Performance (ALoSP) bestimmt und eingehalten werden.

Mit den folgenden Fragen soll nachgegangen werden, ob diese Neugestaltung der Flugsicherheitsaufsicht, Ihrer Einschätzung nach, die Gewährleistung hoher Flugsicherheit beeinflusst.

Es geht also grundsätzlich um die Frage, ob hohe Flugsicherheit und wirtschaftlicher Erfolg einander anspornen, oder einander im Weg stehen.

Beantworten Sie dazu bitte die folgenden Fragen jeweils mit einer Zahl zwischen 1 und 5.

Wobei 1 ,Stimme voll zu<, 2 ,Stimme eher zu<, 3 steils-teils<, 4 ,Stimme eher nicht zu< und 5 ,Stimme gar nicht zu<, bedeutet.

Vielen Dank für Ihre Mitarbeit im Namen der Wissenschaft.

\begin{tabular}{|c|c|c|c|c|}
\hline \multicolumn{5}{|c|}{$\begin{array}{ll}\text { Fragebogen } \\
\end{array}$} \\
\hline \multicolumn{2}{|c|}{$\begin{array}{l}\text { Bei den Werten für Mean und Std. Dev. liegt keine Repräsentativität vor, sie } \\
\text { haben lediglich explorative Bedeutung. }\end{array}$} & $N$ & Mean & $\begin{array}{l}\text { Std. } \\
\text { Dev. }\end{array}$ \\
\hline \multirow{10}{*}{$\begin{array}{l}\text { Ob Flugsicherheit } \\
\text { und Wirtschaftli- } \\
\text { cher Erfolg Hand } \\
\text { in Hand gehen }\end{array}$} & 1. Flugsicherheit zahlt sich finanziell langfristig aus! & 9 & 1,2 & 0,44 \\
\hline & $\begin{array}{l}\text { 2. Flexible internationale Flugsicherheitsstandards wür- } \\
\text { den Luftfahrtgesellschaften wettbewerbsfähiger } \\
\text { machen! }\end{array}$ & 8 & 3,3 & 1,13 \\
\hline & $\begin{array}{l}\text { 3. Flexible internationale Flugsicherheitsstandards } \\
\text { könnten zu Lasten internationaler Flugsicherheit } \\
\text { ausgenutzt werden! }\end{array}$ & 9 & 1,6 & 1,03 \\
\hline & $\begin{array}{l}\text { 4. Gewinnmaximierung und Flugsicherheitsmaximie- } \\
\text { rung sind gleichzeitig möglich! }\end{array}$ & 9 & 2,9 & 2,11 \\
\hline & $\begin{array}{l}\text { 5. Das Senioritätsprinzip behindert den maximal mög- } \\
\text { lichen wirtschaftlichen Erfolg einer Luftfahrtgesell- } \\
\text { schaft! }\end{array}$ & 9 & 2,1 & 1,61 \\
\hline & Das Senioritätsprinzip fördert Flugsicherheit! & 9 & 3 & 1,5 \\
\hline & $\begin{array}{l}\text { 7. Kontinuierliche Arbeitsintensivierung ist dem wirt- } \\
\text { schaftlichen Erfolg förderlich! }\end{array}$ & 8 & 2,5 & 1,71 \\
\hline & $\begin{array}{l}\text { 8. Kontinuierliche Arbeitsintensivierung ist Flugsicher- } \\
\text { heit abträglich! }\end{array}$ & 8 & 2,9 & 2,7 \\
\hline & $\begin{array}{l}\text { 9. In der Luftfahrtbranche ist heutzutage eher das Wirt- } \\
\text { schaften der Zweck, und die Passagierbeförderung } \\
\text { das Mittel! }\end{array}$ & 9 & 2,9 & 3,61 \\
\hline & $\begin{array}{l}\text { 10. Wettbewerbsbegrenzung würde höherer Flugsicher- } \\
\text { heit dienen! }\end{array}$ & 8 & 2,9 & 2,41 \\
\hline
\end{tabular}


11. Wenn eine Luftfahrtgesellschaft Flugsicherheit unabhängig von den Kosten, also mit immens hohen Kosten verfolgt, ist die Wahrscheinlichkeit, dass sie vom Markt eliminiert wird, heute größer, als vor der Liberalisierung/ Deregulierung der Branche!

12. Befinden Sie sich zuweilen in Situationen, in denen Sie als Führungskraft Entscheidungen treffen müssen, bei denen Sie einen Druck zu einer Entscheidung für die kostengünstigere Variante verspüren, auch wenn diese Entscheidung flugsicherheitsrelevant bedenklich, oder Ihrem persönlichen Verständnis von Flugsicherheit zuwider läuft?

13. Flugsicherheit braucht feste internationale Standards, damit nicht auf Kosten der Flugsicherheit Wettbewerb betrieben wird!

$9 \quad 1,9 \quad 1,61$

$9 \quad 3,2 \quad 2,94$

$9 \quad 1,2 \quad 0,19$

14. Hohe Flugsicherheit sollte verfolgt werden, weil man Menschen befördert, und nicht weil man Reputationskosten vermeiden will!

$9 \quad 1,6 \quad 0,28$

15. Hohe Flugsicherheit wird in der unternehmerischen Praxis auch tatsächlich verfolgt weil man seiner Verantwortung den Menschen gegenüber nachkommen will, und nicht wegen finanzieller Überlegungen!

16. Stimmen Sie dieser Beschreibung von Flugsicherheit zu: «Flugsicherheit ist eine Art bzw. Einstellung, vorausschauend Puffer zu lassen, einen Sicherheitsabstand zu lassen, der bei unvorhergesehenen Ereignissen, als Ausweg dienen kann." ?

17. Flugsicherheit ist kontrovers und eben nicht eindeutig!

Was Flugsicherheit
bedeutet

Wirtschaftliches Umfeld

18. Stimmen Sie dieser Aussage zu: »Flugsicherheit ist ein Prinzip, an dem wir uns in der Luftfahrtbranche orientieren müssen, wenn wir klären wollen, ob wir verantwortungsvoll handeln wollen « ?

$9 \quad 2,2 \quad 1,19$

$9 \quad 2 \quad 1,75$

19. Der "acceptable safety level « muss mit dem Gewissen des Verantwortlichen im Luftfahrtunternehmen vereinbar sein und eben nicht mit der » unbegrenzten Öffentlichkeit «!

20. Stimmen Sie dieser Beschreibung von Rentabilität zu: »Rentabilität ist das Ausrichten aller Entscheidungsprozesse in einem Unternehmen auf Wert-

$7 \quad 2,4 \quad 1,62$
schöpfung, im Sinne steigender Rendite «?

21. Für den wirtschaftlichen Erfolg müssen sich Unternehmen manchmal auf Kosten anderer durchsetzen!

22. Wirtschaftswachstum ist in der heutigen Wirtschaftsordnung notwendige Voraussetzung!

$6 \quad 2 \quad 1,2$

23. Der Kapitalmarkt entspricht einem Markt für Unternehmenskontrolle!

$7 \quad 1,7 \quad 0,57$

$\begin{array}{lll}7 & 3,9 & 0,81\end{array}$

24. Renditesteigerung sollte die schlussendliche Maßgabe eines Unternehmens sein!

$\begin{array}{lll}7 & 3,6 & 0,95\end{array}$

Abbildung 3: Interview-Fragebogen (Quelle: eigene Darstellung - keine Repräsentativität, lediglich explorative Bedeutung) 


\section{Literaturverzeichnis}

Adrangi, B./Chow, G./Raffiee, K. (1997): Airline Deregulation, Safety, and Profitability in the US, in: Transportation Journal, Vol. 36/No. 4, 44-51.

Air Berlin (2013): Geschäftsbericht 2012. Link: http://ir.airberlin.com/de/ir/ finanzberichte/ geschaefts-und-zwischenfinanzberichte/2012 (zuletzt abgerufen am 26.02.2017).

Albach, H. (2005): Betriebswirtschaftslehre ohne Unternehmensethik, in: Zeitschrift für Betriebswirtschaft, Jg. 75/H. 9, 809-831.

Banerjee, S. B. (2007): Corporate Social Responsibility: The Good, the Bad, and the Ugly, in: Critical Sociology, Vol. 34/No. 1, 51-71.

Berkeley, E./Hansen, M./McAndrews, C. (2008): History of Aviation Safety Oversight in the United States, (Eds.): Federal Aviation Administration US Department of Transportation, University of California at Berkeley, Berkeley. Link: http://www.tc.faa.gov/ its/worldpac/ techrpt/ar0839.pdf (zuletzt abgerufen am 26.02.2017).

British Airways (2013): Annual Report 2012. Link: http://www.iairgroup.com/phoenix. zhtml? $\mathrm{c}=240949 \& \mathrm{p}=$ irol-reportsannual (zuletzt abgerufen am 26.02.2017).

Bundesministerium der Justiz (2008): $\$ 3$ Unfallverhütung und Flugsicherheitsprogramm (zu OPS 1.035 und 1.037), 1. DV LuftBO. Link: http://www.gesetze-im-internet.de /luftbodv_1_2008/BJNR508900008.html (zuletzt abgerufen am 26.02.2017).

Busse, C. (2013): Der billige Traum, in: Süddeutsche Zeitung, Nr. 87, 17.

Butterwegge, C./Lösch, B./Ptak, R. (2008): Kritik des Neoliberalismus, 2., verbesserte Aufl., Wiesbaden: VS Verlag.

Button, K. (1991): Airline Deregulation, International Experiences, New York: New York University Press.

Canedo, E. (2008): The Rise of the Deregulation Movement in Modern America, 1957-1980, Department of History, Columbia University, New York.

Carrol, A./Shabana K. M. (2010): The Business Case for Corporate Social Responsibility: A Review of Concepts, Research and Practice, in: International Journal of Management Reviews, Vol. 12/No. 1, 85-105.

Chacin, E. (2013): Introduction of Safety Risk Management and NAM/CAR \& SAM. Regional Examples of Runway Excursions. ICAO, St John's. Link: http://www.icao.int/ Meetings/AntiguaRRSS/Presentations/S7\%20P1\%20SRM\%20and \%20Regional\%20Examples.pdf (zuletzt abgerufen am 26.02.2017).

Chen, K.-W./Hsu, Y.-L./Li, W.-C. (2010): Structuring Critical Success Factors of Airline Safety Management System Using a Hybrid Model, in: Transportation Research Part E46, 222235.

Cokorilo, O./Gvozdenovic, S./Vasov, L./Mirosavljevic, P. (2010): Costs of Unsafety in Aviation, in: Technological and Economic Development of Economy, Vol. 16/No. 2, 188-201.

Commission of the European Communities (2001): Green Paper, Promoting a European framework for Corporate Social Responsibility, Brüssel.

Dionne, G./Gagne, R./Gagnon, F./Vanasse, C. (1997): Debt, Moral Hazard and Airline Safety. An Empirical Evidence, in: Journal of Econometrics, Vol. 79/No. 2, 379-402.

EasyJet (2013): Annual Report 2012. Link: http://corporate.easyjet.com/investors/reports-andpresentations/2012 (zuletzt abgerufen am 26.02.2017).

Europäisches Parlament (2013): New European Implementing Rules for Flight and Duty Time Limitations (FTL) - Mini Hearing 18.06.2013. (Eds.): EU Transport and Tourism Committee, Brussels. Link: http://www.youtube.com/watch?v=ygbUPuuQSXk \&src_vid=rTILDGxBvk\&feature=iv\&annotation_id=annotation_485605 (zuletzt abgerufen am 26.02.2017).

Fleming, P./Jones, M. T. (2013): The End of Corporate Social Responsibility. Crisis and Critique, Los Angeles: Sage.

Friedman, M. (1970): The Social Responsibility of Business is to Increase its Profits, in: The New York Times Magazine, 13. September 1970, The New York Times Company.

Graham, D. R./Bowes, M. (1979): Do Finances Influence Airline Safety, Maintenance, and Services? (CRC-386), in: The Public Research Institute, Alexandria, Virginia.

Haigh, M./Jones, M. T. (2006): The Drivers of Corporate Social Responsibility: A Critical Review, in: The Business Review Cambridge, Vol. 5/No. 2, 245-252. 
Hansen, E. G. (2010): Responsible Leadership Systems. An Empirical Analysis of Integrating Corporate Responsibility into Leadership Systems, Wiesbaden: Gabler.

Hansen, U./Schrader, U. (2005): Corporate Social Responsibility als aktuelles Thema der Betriebswirtschaftslehre, in: Die Betriebswirtschaft, Jg. 65/H. 4, 373-395.

Harris, D. (2011): Rule Fragmentation in the Airworthiness Regulations: A Human Factors Perspective, in: Engineering Psychology and Cognitive Ergonomis, 9th International Conference, EPCE 2011, Held as Part of HCI International 2011, Berlin, Heidelberg: Springer, $546-555$.

Ho, J. C./Qui, M./Tang, X. (2013): Do Airlines always suffer from Crashes?, in: Economic Letters, Vol. 118/No. 1, 113-117.

ICAO (2013): DOC 9859, Link: http://www.icao.int/safety/SafetyManagement/Documents/ Doc.9859.3rd\%20Edition.alltext.en.pdf (zuletzt abgerufen am 27.07.2016).

Karnani, A. (2010): The Case Against Corporate Social Responsibility, in: The Wall Street Journal, 23. August 2010. Link: http://www.wsj.com/articles/SB1000142405274870 3338004575230112664504890 (zuletzt abgerufen am 26.02.2017).

Klompstra, M./Roelen, A./Zmarrou, H. (2012): Aviation Safety Management as a Control Process, Transforming Regulatory Requirements into a Working System, NLR Air Transport Safety Institute, Antwerp.

Kuhn, T./Weibler, J. (2011): Ist Ethik ein Erfolgsfaktor? Unternehmensethik im Spannungsfeld von Oxymoron Case, Business Case und Integrity Case, in: Zeitschrift für Betriebswirtschaft, Jg. 81/H. 1, 93-118.

Kuhn, T./Weibler, J. (2016): Management-Derailment und System-Derailment: Zum Konnex zweier Probleme, in: Organisationsberatung, Supervision, Coaching, Jg. 23/H. 2, 133-146.

Kurucz, E. C./Colbert, B. A./Wheeler, D. (2008): The Business Case for Corporate Social Responsibility, in: Crane, A./McWilliams, A./Matten, D./Moon, J./Siegel, D.S. (Eds.) : The Oxford Handbook of Corporate Social Responsibility, Oxford: University Press, 83-112.

Lamnek, S. (1995a): Qualitative Sozialforschung, Methodologie, Bd. 1, 3. Aufl., München, Weinheim: Psychologie-Verlags-Union.

Lamnek, S. (1995b): Qualitative Sozialforschung, Methoden und Techniken, Bd. 2, 3. Aufl., München, Weinheim: Psychologie-Verlags-Union.

Leisinger, K. M. (2008): Zur Relevanz der Unternehmensethik in der Betriebswirtschaftslehre (oder: The Business of Business is Still Business - but the Rules have Changed), in: Schmalenbachs Zeitschrift für betriebswirtschaftliche Forschung, Sonderheft 58, 26-49.

Lufthansa (2013): Geschäftsbericht 2012. Link: http://investor-relations.lufthansagroup.com/ en/finanzberichte/annual-report/2012.html (zuletzt abgerufen am 26.02.2017).

Mayer, H. O. (2004): Interview und schriftliche Befragung. Entwicklung, Durchführung und Auswertung, 2. Aufl., München, Wien: Oldenbourg.

Mintzberg, H. (1983): The Case for Social Responsibility, in: Journal of Business Strategy, Vol. 4/No. 2, 3-15.

Monk, E. (2006): »It pays to be bad«, FTAdvisor (Financial Times), 29. März. Link: http:// www.ftadviser.com/2011/11/02/investments/it-pays-to-be-bad-OInSsf5JtRcGo1glrRpOtM/article.html (zuletzt abgerufen am 27.07.2016).

NTSB (2002): Alaskan Airlines Flight 261. Link: http://www.ntsb.gov/investigations/ AccidentReports/Reports/AAR0201.pdf (zuletzt abgerufen am 26.02.2017).

Orlitzky, M./Schmidt, F. L./Rynes, S. L. (2003): Corporate Social and Financial Performance: A Meta-analysis, in: Organization Studies, Vol. 24/No. 3, 403-441.

Pompl, W. (2007): Luftverkehr. Eine ökonomische und politische Einführung, 5. Aufl., Berlin: Springer.

Raghavan, S./Rhoades, D. L. (2005): Revisiting the Relationship between Profitability and AirCarrier Safety in the US Airline Industry, in: Journal of Air Transport Management, Vol. 11/No. 4, 283-290.

Raith, D. (2013): Mythos CSR: zur Verwertung von Verantwortung und Beratung, Berlin: Springer.

Reason, J. (1990): The Contribution of Latent Human Failures to the Breakdown of Complex Systems, in: Philosophical Transactions of the Royal Society of London. Series B, Biological Sciences, Vol. 327, 475-484. 
Rose, N. L. (1990): Profitability and Product Quality: Economic Determinants of Airline Safety Performance, in: Journal of Political Economy, Vol. 98/No. 5, 944-964.

RTE (2012): RTE News. Link: http://www.rte.ie/news/2012/0816/333847-ryanair-spain/ (zuletzt abgerufen am 26.02.2017).

Ryanair (2012): Code of Business Conduct and Ethics 2012. Link: http://www.ryanair.com/ doc/investor/2012/code_of_ethics.pdf (zuletzt abgerufen am 26.02.2017).

Ryanair (2013): Annual Report 2012. Link: http://www.ryanair.com/doc/investor/2013/ final_annual_report_2013_130731.pdf (zuletzt abgerufen am 26.02.2017).

Ryanair-Safety (2013): Ryanair Safety. Link: http://www.ihateryanair.org/tag/ryanair-safety/ (zuletzt abgerufen am 18.05.2015).

Schenk, G. P. (2004): Auf dem Weg zu einem gemeinsamen Markt im Luftverkehr. Entwicklung der Liberalisierung und der Verkehrsmärkte in den USA und Europa. Hamburger Universität für Wirtschaft und Politik, Hamburg. Link: http://ediss.sub.uni-hamburg.de/ volltexte/2004/2129/pdf/Dissertation.pdf (zuletzt abgerufen am 26.02.2017).

Steinmann, H./Löhr, A. (1991): Grundlagen der Unternehmensethik, Stuttgart: Poeschel Verlag.

Thielemann, U./Ulrich, P. (2009): Standards guter Unternehmensführung. Zwölf internationale Initiativen und ihr normativer Orientierungsgehalt, Bern, Stuttgart, Wien: Haupt.

Thielemann, U. (2009): System Error. Warum der freie Markt zur Unfreiheit führt, Frankfurt am Main: Westend.

Ulrich, P. (2016): Integrative Wirtschaftsethik. Grundlagen einer lebensdienlichen Ökonomie, 5. Aufl., Bern: Haupt.

Valor, C. (2008): Can Consumers Buy Responsibility: Analysis and Solutions for Market Failures, in: Journal of Consumer Policy, Vol. 31/No. 3, 315-326.

Vogel, D. (2006): The Market for Virtue. The Potential and Limits of Corporate Social Responsibility, Washington: Brooking Institution Press.

Wicker, T. (1976): The Most Pervasive Corruption, in: The New York Times, 19. October 1976, 39.

Zadek, S. (2004): The Path to Corporate Responsibility, in: Harvard Business Review, December, 1-8. 